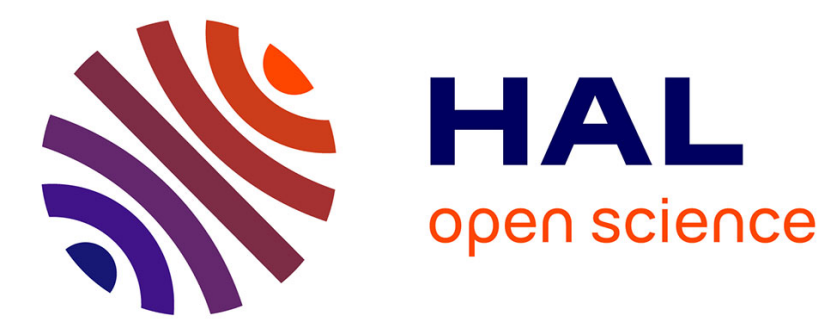

\title{
Lifting particle coordinate changes of magnetic moment type to Vlasov-Maxwell Hamiltonian dynamics
}

Philip Morrison, Michel Vittot, Loïc de Guillebon

\section{To cite this version:}

Philip Morrison, Michel Vittot, Loïc de Guillebon. Lifting particle coordinate changes of magnetic moment type to Vlasov-Maxwell Hamiltonian dynamics. Physics of Plasmas, 2013, 20 (3), pp.032109. 10.1063/1.4794828 . hal-00794557

\section{HAL Id: hal-00794557 \\ https://hal.science/hal-00794557}

Submitted on 26 Feb 2013

HAL is a multi-disciplinary open access archive for the deposit and dissemination of scientific research documents, whether they are published or not. The documents may come from teaching and research institutions in France or abroad, or from public or private research centers.
L'archive ouverte pluridisciplinaire $\mathbf{H A L}$, est destinée au dépôt et à la diffusion de documents scientifiques de niveau recherche, publiés ou non, émanant des établissements d'enseignement et de recherche français ou étrangers, des laboratoires publics ou privés. 


\title{
Lifting particle coordinate changes of magnetic moment type to Vlasov-Maxwell Hamiltonian dynamics
}

\author{
P. J. Morrison* \\ Department of Physics and Institute for Fusion Studies, \\ University of Texas at Austin, Austin, TX 78712, USA \\ M. Vittot ${ }^{\dagger}$ and L. de Guillebon ${ }^{\ddagger}$ \\ Centre de Physique Theorique \\ Aix-Marseille Universite, CNRS, CPT \\ UMR 7332, 13288 Marseille, France \\ and \\ Universite de Toulon, CNRS, CPT \\ UMR 7332, 83957 La Garde, France
}

\begin{abstract}
Techniques for coordinate changes that depend on both dependent and independent variables are developed and applied to the Maxwell-Vlasov Hamiltonian theory. Particle coordinate changes with a new velocity variable dependent on the magnetic field, with spatial coordinates unchanged, are lifted to the field theoretic level, by transforming the noncanonical Poisson bracket and Hamiltonian structure of the Vlasov-Maxwell dynamics. Several examples are given including magnetic coordinates, where the velocity is decomposed into components parallel and perpendicular to the local magnetic field, and the case of spherical velocity coordinates. An example of the lifting procedure is performed to obtain a simplified version of gyrokinetics, where the magnetic moment is used as a coordinate and the dynamics is reduced by elimination of the electric field energy in the Hamiltonian.
\end{abstract}

Key Words: Maxwell-Vlasov, Hamiltonian, noncanonical Poisson bracket, lift, magnetic moment, guiding-center reduction, gyrokinetics.

\footnotetext{
* morrison@physics.utexas.edu

$\dagger$ michel.vittot@cpt.univ-mrs.fr

$\ddagger$ loic.de-guillebon@cpt.univ-mrs.fr
} 


\section{INTRODUCTION}

Perturbation theory in the context of Hamiltonian dynamics has proved to be unquestionably useful in many contexts, ranging from celestial mechanics (e.g. [1]), to atomic physics (e.g. [2]), to plasma physics (e.g. [3]). The superconvergent expansions of KolmogorovArnold-Moser theorem (e.g. [4]) and the techniques of adiabatic invariance (e.g. [5]) all are aspects of perturbation theory in the Hamiltonian context. Although such techniques are well-developed and well-known for finite-dimensional systems, this is not the case for such perturbation theories for partial differential equations. This is particularly true for Hamiltonian systems with noncanonical Poisson brackets of the form of those given in [6-8] for plasma systems. A main goal of the present paper is to provide tools for such perturbation theory using the Poisson bracket for Vlasov-Maxwell equations [7, 9-12] in situations with a short time scale introduced by the presence of a strong magnetic field.

Derivations of gyrokinetic theories have proceeded directly from the Vlasov-Maxwell equations of motion as in the nonlinear development of [13], they have been based on Hamiltonian particle orbit perturbation theory that is lifted up to the kinetic level as in the linear development of [14], or they have incorporated both particle orbit and kinetic perturbations to arrive at a nonlinear theory [15]. (See [16, 17] for review.) None of these procedures parallels that for finite-dimensional Hamiltonian systems that has historically achieved such great success. Consequently, none of these theories obtain an infinite-dimensional Hamiltonian form as a consequence of their method of derivation. In fact, at present it is not known if nonlinear gyrokinetics has Hamiltonian form, the form possessed by all of the important systems of plasma physics when dissipative terms are neglected. (For review of Hamiltonian structure and techniques see $[7,8,18]$ ). An alternative approach was recently introduced in [19], one which uses a kind of kinetic action principle (see, e.g., [20]), rather than the Hamiltonian structure of the Vlasov-Maxwell dynamics, and one that incorporates an approximatly self-consistent electromagnetic field.

To effect an infinite-dimensional Hamiltonian gyrokinetic-like perturbation theory requires changes of particle coordinates, motivated by the near constancy of the magnetic moment, that induce changes in the field Hamiltonian dynamics. Thus, this perturbation theory involves a sequence of particle coordinate changes that depends on the dependent (field) variables and their arguments, which are independent variables from the point of 
view of the Hamiltonian structure. This complicates matters significantly and care must be taken when performing transformations, most notably with the chain rule. Because the Vlasov-Maxwell theory has fields of mixed type, the electromagnetic fields depending on a space variable and the distribution function depending on a phase space variable, and because these fields are not a usual canonical set, the situation is further complicated. In the present paper the intricacies of this kind of transformation and associated chain rule are described, which enables the Hamiltonian perturbation theory. The techniques are then applied to obtain a simplified version of gyrokinetics (guiding center kinetics), which considers the presence of a conserved magnetic moment, as a first step for more general gyrokinetic reduction, e.g. by using the intrinsic coordinates developed in [21], that will be considered elsewhere.

We refer to the process of determining the form of field dynamics, induced by particle orbit dynamics, as lifting. Lifting in the present context is a natural relative of that treated in Ref. [12], a companion paper that treats the lifting of microscopic particle dynamics up to the field level. Actually, the purpose of the present paper is distinct from that of Ref. [12], but the framework is closely related. Reference [12] is about a general prescription for the Hamiltonian structure of Vlasov-Maxwell theories with polarization and magnetization, but often such effects come from a transformation of the particle phase-space. On the other hand, here, we study how to lift a change of particle coordinates to the Hamiltonian structure of Vlasov-Maxwell dynamics. As a side effect, this can generate polarization and magnetization and fits in with the purpose of Ref. [12]. Thus, the two papers study complementary aspects of one and the same question.

The paper is organized as follows. In Sec. II some preliminary material needed for the subsequent development is described. This is followed by four sections where several specific transformations are considered, and it is shown how to lift these coordinate transformations, which are tailored to particle orbit dynamics, up to the level of fields, by detailing how to transform the Vlasov-Maxwell Poisson bracket into the new coordinates. In the process, four features associated with steps of lifting must be considered; these are progressively taken into account to reach the final complete transformation for the magnetic moment. The four features/steps are

Step A: A chain rule for functions in the particle bracket $[\cdot, \cdot]$, because the transformation affects particle coordinates. 
Step B: The presence of the Jacobian both in functionals and in functional derivatives.

Step C: A chain rule for functionals in the field bracket $\{\cdot, \cdot\}$, because the transformation depends of the magnetic field, which is a dynamical field.

Step D: The possible presence of gradients of the magnetic field in the transformation, which implies the presence of differential operators in the chain rule.

Section III considers magnetic coordinates, where the particle velocity coordinate is projected parallel and perpendicular to a space-dependent dynamic magnetic field; this introduces the features A and B mentioned above. Next, in Sec. IV, spherical velocity coordinates are considered. Here the velocity coordinates are chosen as the unit vector of the velocity (independent of the spatial coordinates) and a coordinate in one-to-one correspondence with the norm of the velocity. This transformation introduces a new feature in that the Jacobian determinant of the transformation is no longer unity, but the transformation does not depend of the magnetic field. This provides a simple example for the features A and C. In Sec. V, we turn to a case that is both more complete and closer to that needed for gyrokinetics with the magnetic moment coordinate: the change of coordinates depends on the local value of the magnetic field, which implies the presence of the features A, B and C. Next, Sec. VI considers the physically important situation, where the change of coordinates involves spatial derivatives of the magnetic field to arbitrary order, i.e., as given by Eq. (1) below; this involves all of the four features A, B, C, and D. With the techniques of the previous four sections in hand, in Sec. VII we treat an example where the reduced coordinate is indeed the magnetic moment and explicitly transform the Hamiltonian form of the Vlasov-Maxwell equation into the new coordinates. Finally, in Sec. VIII, we conclude.

Notice that the first example differs from the others in that the last four sections IVVII are about the same ultimate transformation, with each section bringing us closer to the magnetic moment transformation: the velocity coordinates are first chosen as spherical coordinates; then the norm of the velocity is taken as a general local function of the magnetic field; next, it is taken as a general non-local function of the magnetic field; last, it is chosen as the magnetic moment. Also, for the sake of conciseness, the equations of motion of the transformed Vlasov-Maxwell system will be studied only in the example of Sec. VII, but it is clear that the conclusions are general and hold for other examples of lifting. 


\section{PRELIMINARY MATERIAL}

From a general point of view, a main purpose of this paper is to transform the VlasovMaxwell Hamiltonian structure when the phase space variables $(\mathbf{q}, \mathbf{v})$ are changed to the following new coordinates that depend on the magnetic field and all of its derivatives:

$$
\overline{\mathbf{q}}=\mathbf{q}, \quad \overline{\mathbf{v}}=\overline{\mathbf{v}}(\mathbf{q}, \mathbf{v} ; \mathbf{B}, \nabla \mathbf{B}, \ldots)
$$

For the noncanonical Hamiltonian structure of Vlasov-Maxwell dynamics, the observables are the set of all functionals of the magnetic field $\mathbf{B}(\mathbf{q})$, the electric field $\mathbf{E}(\mathbf{q})$, and the phase space density $f(\mathbf{q}, \mathbf{v})$, where the time variable has been suppressed. The Poisson bracket is $[7,9-11]$ :

$$
\begin{aligned}
\{F, G\} & =\int d^{3} q d^{3} v f\left[F_{f}, G_{f}\right] \\
& +e \int d^{3} q d^{3} v f\left(G_{\mathbf{E}} \cdot \partial_{\mathbf{v}} F_{f}-F_{\mathbf{E}} \cdot \partial_{\mathbf{v}} G_{f}\right) \\
& +\int d^{3} q\left(F_{\mathbf{E}} \cdot \nabla \times G_{\mathbf{B}}-G_{\mathbf{E}} \cdot \nabla \times F_{\mathbf{B}}\right),
\end{aligned}
$$

where subscripts are used for functional derivatives, $F_{f}:=\delta F / \delta f, F_{\mathbf{E}}:=\delta F / \delta \mathbf{E}$, etc., and the particle bracket is $[g, h]=\nabla g \cdot \partial_{\mathbf{v}} h-\nabla h \cdot \partial_{\mathbf{v}} g+e \mathbf{B} \cdot \partial_{\mathbf{v}} g \times \partial_{\mathbf{v}} h$, with $\nabla g=\partial g / \partial \mathbf{q}$ and $\partial_{\mathbf{v}} h=\partial h / \partial \mathbf{v}$, for any functions of the phase-space $g(\mathbf{q}, \mathbf{v})$ and $h(\mathbf{q}, \mathbf{v})$. For the sake of simplicity physical constants have been scaled away as usual, but a dimensionless charge variable $e$ that indicates the coupling term has been retained (see [12] for a dimensional form of this bracket). The variable $e$ becomes the charge ratios when (2) is generalized by summing over multiple species.

The Hamiltonian functional is

$$
H[\mathbf{E}, \mathbf{B}, f]=\frac{1}{2} \int d^{3} q d^{3} v\|\mathbf{v}\|^{2} f+\frac{1}{2} \int d^{3} q\left(\|\mathbf{E}\|^{2}+\mid \mathbf{B} \|^{2}\right),
$$

which is the sum of the kinetic energy of the plasma and the energy of the electromagnetic field. The relativistic model is obtained by replacing $\|\mathbf{v}\|^{2}$ in the kinetic energy term with $\sqrt{1+\|\mathbf{v}\|^{2}}$, where in the latter case $\mathbf{v}$ is the scaled relativistic momentum. The coupling between the plasma and electromagnetic field is included in the noncanonical Poisson bracket (2). The Hamiltonian (3) together with the Poisson bracket generates the motion through Hamilton's equations expressed as

$$
\dot{F}=\{F, H\}
$$


for any observable $F$. In particular, if $F$ denotes the field variables the bracket induces Maxwell-Vlasov equations as follows:

$$
\begin{aligned}
& \dot{\mathbf{B}}=\{\mathbf{B}, H\}=-\nabla \times \mathbf{E}, \\
& \dot{\mathbf{E}}=\{\mathbf{E}, H\}=\nabla \times \mathbf{B}-e \int d^{3} v f \mathbf{v}, \\
& \dot{f}=\{f, H\}=-\mathbf{v} \cdot \nabla f-e(\mathbf{E}+\mathbf{v} \times \mathbf{B}) \cdot \partial_{\mathbf{v}} f .
\end{aligned}
$$

As noted in Sec. I, in order to transform the Hamiltonian structure to facilitate the separation or removal of fast time scales (as in oscillating-center, guiding-center, and gyrokinetic theories) care must be taken because such a change of coordinates involves both the dependent and independent variables, i.e., the spatial observation points of the field. A simple case of this is treated in the next section.

\section{LIFTING WITH MAGNETIC COORDINATES}

As a first case of lifting, consider velocity coordinates based on a decomposition of the velocity using the magnetic field. This transformation of the spatial coordinate is unchanged, but the velocity $\mathbf{v}$ is transformed as follows:

$$
\mathbf{v}=\mathbf{v}(\overline{\mathbf{v}} ; \mathbf{B})=\mathbf{v}\left(v_{\|}, \mathbf{v}_{\perp} ; \mathbf{B}\right)=\hat{\mathbf{b}} v_{\|}+\mathbf{v}_{\perp}
$$

where $\hat{\mathbf{b}}=\mathbf{B} /\|\mathbf{B}\|$ is the unit vector the direction of the magnetic field,

$$
v_{\|}=\hat{\mathbf{b}} \cdot \mathbf{v}
$$

is the (scalar) component of the velocity parallel to the magnetic field, and

$$
\mathbf{v}_{\perp}=\mathbf{v}-\hat{\mathbf{b}} \hat{\mathbf{b}} \cdot \mathbf{v}=\overline{\bar{I}}_{\perp} \cdot \mathbf{v}
$$

is the (vectorial) component of the velocity perpendicular to the magnetic field, with

$$
\overline{\bar{I}}_{\perp}:=\overline{\bar{I}}-\hat{\mathbf{b}} \hat{\mathbf{b}}
$$

being the orthogonal projector onto the plane perpendicular to the magnetic field.

There are two chain rules to consider: that for functions, considered next, and that for functionals, such as the energy expression of (3), which will follow. 


\section{A. Function chain rule}

The transformation of the field Poisson bracket of (2) requires the transformation of the particle bracket,

$$
[g, h]=\frac{\partial g}{\partial \mathbf{q}} \cdot \frac{\partial h}{\partial \mathbf{v}}-\frac{\partial h}{\partial \mathbf{q}} \cdot \frac{\partial g}{\partial \mathbf{v}}+e \mathbf{B} \cdot\left(\frac{\partial g}{\partial \mathbf{v}} \times \frac{\partial h}{\partial \mathbf{v}}\right),
$$

into the new coordinates, $(\mathbf{q}, \mathbf{v}) \rightarrow\left(\mathbf{q}, v_{\|}, \mathbf{v}_{\perp}\right)$. The following abbreviations are convenient:

$$
\nabla:=\frac{\partial}{\partial \mathbf{q}}, \quad \partial_{i}:=\frac{\partial}{\partial q_{i}}, \quad \partial_{\|}:=\frac{\partial}{\partial v_{\|}}, \quad \partial_{\perp}:=\frac{\partial}{\partial \mathbf{v}_{\perp}} .
$$

Note the last operator acts only in the plane perpendicular to $\mathbf{B}$, which implies the following properties:

$$
\partial_{\perp} \bar{g} \cdot \overline{\bar{I}}_{\perp}=\partial_{\perp} \bar{g} \quad \text { and } \quad \hat{\mathbf{b}} \cdot \partial_{\perp} \bar{g}=0 .
$$

Here and everywhere in the paper, the overbar over a function will indicate a function of the phase-space expressed in new coordinates $\bar{g}(\mathbf{q}, \overline{\mathbf{v}})$.

Total variations of $g(\mathbf{q}, \mathbf{v})=\bar{g}\left(\mathbf{q}, v_{\|}, \mathbf{v}_{\perp}\right)$ are given by

$$
\delta g=\frac{\partial g}{\partial \mathbf{q}} \cdot \delta \mathbf{q}+\frac{\partial g}{\partial \mathbf{v}} \cdot \delta \mathbf{v}=\nabla \bar{g} \cdot \delta \mathbf{q}+\partial_{\|} \bar{g} \cdot \delta v_{\|}+\partial_{\perp} \bar{g} \cdot \delta \mathbf{v}_{\perp}
$$

while variations of the initial and final coordinates are related by

$$
\begin{aligned}
\delta v_{\|}= & \hat{\mathbf{b}} \cdot \delta \mathbf{v}+(\delta \mathbf{q} \cdot \nabla \hat{\mathbf{b}}) \cdot \mathbf{v} \\
\delta \mathbf{v}_{\perp}= & \overline{\bar{I}}_{\perp} \cdot \delta \mathbf{v}+\delta \overline{\bar{I}}_{\perp} \cdot \mathbf{v} \\
= & \overline{\bar{I}}_{\perp} \cdot \delta \mathbf{v}-(\delta \mathbf{q} \cdot \nabla \hat{\mathbf{b}})(\hat{\mathbf{b}} \cdot \mathbf{v}) \\
& \quad \hat{\mathbf{b}}(\delta \mathbf{q} \cdot \nabla \hat{\mathbf{b}}) \cdot \mathbf{v} .
\end{aligned}
$$

For the function chain rule the field $\mathbf{B}$ is assumed to be a fixed function with the coordinates $(\mathbf{q}, \mathbf{v})$ changing.

Inserting (7) into (6) implies the chain rule relations

$$
\begin{aligned}
& \frac{\partial g}{\partial \mathbf{v}}=\hat{\mathbf{b}} \partial_{\|} \bar{g}+\partial_{\perp} \bar{g} \cdot \overline{\bar{I}}_{\perp}=\hat{\mathbf{b}} \partial_{\|} \bar{g}+\partial_{\perp} \bar{g} \\
& \frac{\partial g}{\partial q_{i}}=\partial_{i} \bar{g}+\left(\mathbf{v} \cdot \partial_{i} \hat{\mathbf{b}}\right) \partial_{\|} \bar{g}-(\hat{\mathbf{b}} \cdot \mathbf{v}) \partial_{\perp} \bar{g} \cdot \partial_{i} \hat{\mathbf{b}}
\end{aligned}
$$

and using (8) and (9) in (5) gives the particle bracket in the magnetic coordinates

$$
\begin{aligned}
{[\bar{g}, \bar{h}] } & =\hat{\mathbf{b}} \cdot\left(\nabla \bar{g} \partial_{\|} \bar{h}-\nabla \bar{h} \partial_{\|} \bar{g}\right) \\
& +\left(\nabla \bar{g} \cdot \partial_{\perp} \bar{h}-\nabla \bar{h} \cdot \partial_{\perp} \bar{g}\right) \\
& +\mathbf{a} \cdot\left(\partial_{\perp} \bar{h} \partial_{\|} \bar{g}-\partial_{\perp} \bar{g} \partial_{\|} \bar{h}\right) \\
& +\partial_{\perp} \bar{g} \cdot \overline{\bar{b}} \cdot \partial_{\perp} \bar{h}+e \mathbf{B} \cdot\left(\partial_{\perp} \bar{g} \times \partial_{\perp} \bar{h}\right),
\end{aligned}
$$


with

$$
a_{i}=\mathbf{v} \cdot \partial_{i} \hat{\mathbf{b}}+(\hat{\mathbf{b}} \cdot \mathbf{v}) \hat{\mathbf{b}} \cdot \nabla \hat{\mathbf{b}}_{i} \quad \text { and } \quad \overline{\bar{b}}_{i j}=(\hat{\mathbf{b}} \cdot \mathbf{v})\left(\partial_{i} \hat{\mathbf{b}}_{j}-\partial_{j} \hat{\mathbf{b}}_{i}\right)
$$

In all these relations, recall that $\partial_{\perp} \bar{g}=\partial_{\perp} \bar{g} \cdot \overline{\bar{I}}_{\perp}$. This is important because, for instance, the component of $\nabla \bar{g}$, a or $\overline{\bar{b}}$ parallel to $\hat{\mathbf{b}}$ are non-zero, but vanish when contracted with $\partial_{\perp} \bar{g}$

\section{B. Jacobian}

In general care must be taken with the Jacobian determinant $\mathcal{J}$ when defining functional derivatives, but here Step B is trivial, since the Jacobian is unity

$$
\mathcal{J}:=\frac{\partial\left(\mathbf{q}, v_{\|}, \mathbf{v}_{\perp}\right)}{\partial(\mathbf{q}, \mathbf{v})}=1
$$

This follows because rotations have unit Jacobians and at any time there exists a rotation to a cartesian coordinate system with one of the $\mathbf{v}$ axes aligned with $\hat{\mathbf{b}}$. Thus

$$
d z:=d^{3} q d^{3} v=d^{3} q d v_{\|} d^{2} v_{\perp}=: d q d v .
$$

Because the volume integral is ultimately independent of how it is calculated, $d z$ can be assumed to be independent of $\mathbf{B}$, e.g. when calculating functional derivatives with respect to $\mathbf{B}$, the topic considered next.

\section{Functional chain rule}

For the functional chain rule, the transformation of the fields must be made definite, Here,

$$
\begin{aligned}
\mathbf{E}(\mathbf{q}) & =\overline{\mathbf{E}}(\mathbf{q}), \quad \mathbf{B}(\mathbf{q})=\overline{\mathbf{B}}(\mathbf{q}) \\
f(\mathbf{q}, \mathbf{v}) & =\bar{f}\left(\mathbf{q}, v_{\|}, \mathbf{v}_{\perp}\right)=\bar{f}\left(\mathbf{q}, \hat{\mathbf{b}} \cdot \mathbf{v}, \overline{\bar{I}}_{\perp} \cdot \mathbf{v}\right) \\
& =f\left(\mathbf{q}, \hat{\mathbf{b}} v_{\|}+\mathbf{v}_{\perp}\right),
\end{aligned}
$$

where now the coordinates $(\mathbf{q}, \mathbf{v})$ are fixed and the field $\hat{\mathbf{b}}$ varies.

Variation of a transformed functional, $F[f, \mathbf{B}, \mathbf{E}]=\bar{F}[\bar{f}, \overline{\mathbf{B}}, \overline{\mathbf{E}}]$, gives

$$
\begin{aligned}
\delta F & =\int d z F_{f} \delta f+\int d q\left(F_{\mathbf{B}} \cdot \delta \mathbf{B}+F_{\mathbf{E}} \cdot \delta \mathbf{E}\right) \\
& =\int d z \bar{F}_{\bar{f}} \delta \bar{f}+\int d q\left(\bar{F}_{\overline{\mathbf{B}}} \cdot \delta \overline{\mathbf{B}}+\bar{F}_{\overline{\mathbf{E}}} \cdot \delta \overline{\mathbf{E}}\right) .
\end{aligned}
$$


With the variations of the initial and final fields related by

$$
\delta \mathbf{E}=\delta \overline{\mathbf{E}}, \quad \delta \mathbf{B}=\delta \overline{\mathbf{B}}, \quad \text { and } \quad \delta f=\delta \bar{f}+\partial_{\|} \bar{f}(\mathbf{v} \cdot \delta \hat{\mathbf{b}})+\partial_{\perp} \bar{f} \cdot \delta \overline{\bar{I}}_{\perp} \cdot \mathbf{v}
$$

expressions relating functional derivatives of new and old variables can be obtained. Using

$$
\delta \overline{\bar{I}}_{\perp}=-\frac{1}{\|\mathbf{B}\|}\left(\hat{\mathbf{b}} \overline{\bar{I}}_{\perp} \cdot \delta \mathbf{B}+\overline{\bar{I}}_{\perp} \cdot \delta \mathbf{B} \hat{\mathbf{b}}\right)
$$

and after some work the last equation of (12) becomes

$$
\delta f=\delta \bar{f}+\frac{\left(\mathbf{v}_{\perp} \cdot \delta \mathbf{B}\right)}{\|\mathbf{B}\|} \partial_{\|} \bar{f}-\frac{v_{\|}}{\|\mathbf{B}\|} \delta \mathbf{B} \cdot \partial_{\perp} \bar{f}
$$

Inserting this and the other two equations of (12) into (11), and then equating coefficients, gives the functional chain rule relations

$$
\begin{aligned}
& \frac{\delta F}{\delta f}=\frac{\delta \bar{F}}{\delta \bar{f}}, \quad \frac{\delta F}{\delta \mathbf{E}}=\frac{\delta \bar{F}}{\delta \overline{\mathbf{E}}}, \\
& \frac{\delta F}{\delta \mathbf{B}}=\frac{\delta \bar{F}}{\delta \overline{\mathbf{B}}}+\frac{1}{\|\mathbf{B}\|} \int d v \frac{\delta F}{\delta \bar{f}} \partial_{\mathbf{v}}^{*} \bar{f},
\end{aligned}
$$

where

$$
\partial_{\mathbf{v}}^{*}:=\mathbf{v}_{\perp} \partial_{\|}-v_{\|} \partial_{\perp}
$$

Finally, the Maxwell-Vlasov bracket expressed in these magnetic coordinates is

$$
\begin{aligned}
\{F, G\}= & \int d z f\left[F_{f}, G_{f}\right] \\
+ & e \int d z f\left(G_{\mathbf{E}} \cdot \partial_{\mathbf{v}} F_{f}-F_{\mathbf{E}} \cdot \partial_{\mathbf{v}} G_{f}\right) \\
+ & \int d^{3} q\left(F_{\mathbf{E}} \cdot \nabla \times\left[G_{\mathbf{B}}+\frac{1}{\|\mathbf{B}\|} \int d v G_{f} \partial_{\mathbf{v}}^{*} f\right]\right. \\
& \left.-G_{\mathbf{E}} \cdot \nabla \times\left[F_{\mathbf{B}}+\frac{1}{\|\mathbf{B}\|} \int d v F_{f} \partial_{\mathbf{v}}^{*} f\right]\right),
\end{aligned}
$$

where the 'bars' have been dropped, [, ] means the bracket of (5) rewritten in the new coordinates as (10), and $\partial_{\mathbf{v}}=\hat{\mathbf{b}} \partial_{\|}+\partial_{\perp}$ is a shorthand as in (8). Note, $\partial_{\mathbf{v}}^{*} v^{2}=0$.

\section{LIFTING SPHERICAL VELOCITY COORDINATES $\mathbf{v}=V \hat{\mathbf{v}}$}

Now turn to the new coordinates considered for intrinsic gyrokinetics (used in [21]), which changes only one of the velocity coordinates to get the magnetic moment. The two 
other velocity coordinates are usually chosen as the unit vector of the velocity. So, a preliminary change of coordinates consists in adopting spherical coordinates for the velocity space: $\mathbf{v}=V \hat{\mathbf{v}}$ where $V:=\|\mathbf{v}\| \in \mathbb{R}_{+}$is the norm of the velocity and $\hat{\mathbf{v}}:=\mathbf{v} /\|\mathbf{v}\| \in S^{2}$ is the unit vector of the velocity. This transformation is considered in this section, but later the change $V \rightarrow \mu$ will be considered.

Step A: The transformation $\mathbf{v} \leftrightarrow(\hat{\mathbf{v}}, V)$ is clearly invertible. For the chain rule the following are needed:

$$
\delta V=\hat{\mathbf{v}} \cdot \delta \mathbf{v} \quad \text { and } \quad \delta \hat{\mathbf{v}}=\overline{\overline{\mathcal{I}}}_{\perp} \cdot \frac{\delta \mathbf{v}}{V}
$$

where

$$
\overline{\overline{\mathcal{I}}}_{\perp}=\overline{\bar{I}}-\hat{\mathbf{v}} \hat{\mathbf{v}}
$$

is the orthogonal projector onto the plane perpendicular to the velocity. Note $\overline{\overline{\mathcal{I}}}_{\perp}$ is different from the magnetic projector $\overline{\bar{I}}_{\perp}$ of (4) used in Sec. III.

As in Sec. III the above are used to calculate the function chain rule, giving

$$
\begin{aligned}
& \frac{\partial g}{\partial \mathbf{v}}=\frac{1}{V} \frac{\partial \bar{g}}{\partial \hat{\mathbf{v}}} \cdot \overline{\overline{\mathcal{I}}}_{\perp}+\frac{\partial \bar{g}}{\partial V} \hat{\mathbf{v}}, \\
& \nabla g=\frac{\partial g}{\partial \mathbf{q}}=\frac{\partial \bar{g}}{\partial \mathbf{q}}=\nabla \bar{g} .
\end{aligned}
$$

Inserting (16) and (17) into (5) and, after some manipulations, the particle bracket expressed in spherical coordinates is obtained

$$
\begin{aligned}
{[g, h] } & =\frac{1}{V}\left(\nabla g \cdot \overline{\overline{\mathcal{I}}}_{\perp} \cdot \partial_{\hat{\mathbf{v}}} h-\nabla h \cdot \overline{\overline{\mathcal{I}}}_{\perp} \cdot \partial_{\hat{\mathbf{v}}} g\right) \\
& +\hat{\mathbf{v}} \cdot\left(\nabla g \partial_{V} h-\nabla h \partial_{V} g\right) \\
& +\frac{e \mathbf{B}}{V^{2}} \cdot\left(\partial_{\hat{\mathbf{v}}} g \cdot \overline{\overline{\mathcal{I}}}_{\perp}\right) \times\left(\partial_{\hat{\mathbf{v}}} h \cdot \overline{\overline{\mathcal{I}}}_{\perp}\right) \\
& +\frac{e \mathbf{B} \times \hat{\mathbf{v}}}{V} \cdot\left(\partial_{V} g \partial_{\hat{\mathbf{v}}} h-\partial_{\hat{\mathbf{v}}} g \partial_{V} h\right),
\end{aligned}
$$

where, for convenience, the 'bars' have been dropped and the abbreviations

$$
\frac{\partial g}{\partial \hat{\mathbf{v}}}=: \partial_{\hat{\mathbf{v}}} g \quad \text { and } \quad \frac{\partial g}{\partial V}=: \partial_{V} g
$$

have been employed.

Step B: This step is not trivial here, since the Jacobian for this special case is not unity

$$
d z=V^{2} d V d \Omega d^{3} q=\mathcal{J} d V d \Omega d^{3} q=: \mathcal{J} d \eta d^{3} q=: \mathcal{J} d w
$$


because the integration measures are changed from $d^{3} v$ and $d z$ to $d \eta$ and $d w$, which are defined by relation (19).

Step C: Turning to the functional chain rule, notice that the change of coordinates does not depend on the fields, and the Step C is simplified here, but it is not completely trivial, because the Jacobian has to be taken into account

$$
\begin{aligned}
\delta F & =\int d z F_{f} \delta f+\int d^{3} q\left(F_{\mathbf{B}} \cdot \delta \mathbf{B}+F_{\mathbf{E}} \cdot \delta \mathbf{E}\right) \\
& =\int d w \bar{F}_{\bar{f}} \delta \bar{f}+\int d^{3} q\left(\bar{F}_{\overline{\mathbf{B}}} \cdot \delta \overline{\mathbf{B}}+\bar{F}_{\overline{\mathbf{E}}} \cdot \delta \overline{\mathbf{E}}\right) .
\end{aligned}
$$

Inserting (19) into (20) gives

$$
F_{f}=\mathcal{J}^{-1} \bar{F}_{\bar{f}}, \quad F_{\mathbf{B}}=\bar{F}_{\overline{\mathbf{B}}}, \quad \text { and } \quad F_{\mathbf{E}}=\bar{F}_{\overline{\mathbf{E}}} .
$$

Note, in (21) the new functional derivative is defined with respect to the bare measure $d w$.

So, the first term of the Maxwell-Vlasov bracket transforms as

$$
\{F, G\}_{1}:=\int d z f\left[F_{f}, G_{f}\right]=\int d w \mathcal{J} \bar{f}\left[\mathcal{J}^{-1} \bar{F}_{\bar{f}}, \mathcal{J}^{-1} \bar{G}_{\bar{f}}\right]=\{\bar{F}, \bar{G}\}_{1},
$$

with the bracket of the second equality above given by (18).

In practical computations with Vlasov-like Poisson brackets (especially when computing the equations of motion), a very useful property is the usual ' $f$ - $g$ - $h$ ' identity, viz. $\int d z f[g, h]=-\int d z g[f, h]$, for canonical brackets. Here, because of the Jacobian, this identity is changed and replaced by:

$$
\int d w \mathcal{J} f\left[\mathcal{J}^{-1} g, h\right]=-\int d w g[f, h]
$$

and in terms of the bare measure

$$
\frac{\delta f(w)}{\delta f\left(w^{\prime}\right)}=\delta\left(w-w^{\prime}\right) .
$$

With this identity (23), it is straightforward to verify that the bracket of (22) produces the correct equations of motion for the Vlasov-Poisson system.

Now consider the coupling terms of the bracket

$$
\begin{aligned}
\{F, G\}_{2} & :=e \int d z f\left(G_{\mathbf{E}} \cdot \partial_{\mathbf{v}} F_{f}-F_{\mathbf{E}} \cdot \partial_{\mathbf{v}} G_{f}\right) \\
& =e \int d w \mathcal{J} \bar{f}\left(G_{\overline{\mathbf{E}}} \cdot \partial_{\mathbf{v}} \mathcal{J}^{-1} \bar{F}_{\bar{f}}-F_{\overline{\mathbf{E}}} \cdot \partial_{\mathbf{v}} \mathcal{J}^{-1} \bar{G}_{\bar{f}}\right)
\end{aligned}
$$


where $\partial_{\mathbf{v}}$ is a shorthand for the expression of (16). When generating Maxwell's equations, the Hamiltonian gives

$$
\bar{H}_{\bar{f}}=\mathcal{J}\|\mathbf{v}\|^{2} / 2
$$

which gives the correct expression for the current density $\mathbf{J}=\int d \eta \mathcal{J} f \mathbf{v}$.

Finally, the pure field terms of the Maxwell-Vlasov bracket are unchanged and, thus, the Maxwell-Vlasov bracket in these spherical coordinates becomes

$$
\begin{aligned}
\{F, G\} & =\int d w \mathcal{J} f\left[\mathcal{J}^{-1} F_{f}, \mathcal{J}^{-1} G_{f}\right] \\
& +e \int d w \mathcal{J} f\left(G_{\mathbf{E}} \cdot \partial_{\mathbf{v}} \mathcal{J}^{-1} F_{f}-F_{\mathbf{E}} \cdot \partial_{\mathbf{v}} \mathcal{J}^{-1} G_{f}\right) \\
& +\int d^{3} q\left(F_{\mathbf{E}} \cdot \nabla \times G_{\mathbf{B}}-G_{\mathbf{E}} \cdot \nabla \times F_{\mathbf{B}}\right),
\end{aligned}
$$

where the 'bars' have been dropped, and [, ] means the bracket of (5) rewritten in the new coordinates as (18).

\section{LIFTING WITH LOCAL DEPENDENCE ON B}

To include the magnetic moment in the coordinates, the next step is to investigate the coordinate transformation $V \leftrightarrow A$, where $A$ is a coordinate in one-to-one correspondence with the coordinate $V$ of Sec. IV, but in this section it is assumed to have local dependence on the magnetic field, i.e, it depends on $\mathbf{B}$ but not its derivatives. Explicitly, the transformation is $(\mathbf{q}, V, \hat{\mathbf{v}}) \leftrightarrow(\overline{\mathbf{q}}, A, \hat{\mathbf{w}})$ where

$$
\mathbf{q}=\overline{\mathbf{q}}, \quad \hat{\mathbf{v}}=\hat{\mathbf{w}}, \quad \text { and } \quad V=V(A, \hat{\mathbf{w}}, \mathbf{B}) .
$$

For an example of an allowable function $V$, see Sec. VII. Clearly, invertibility requires $V_{A}:=\partial V / \partial A \neq 0$, which is the only assumption on this function, beside the absence of gradients of the magnetic field B. Since the first two equations above are identities, eventually $\hat{\mathbf{v}}$ will be used for $\hat{\mathbf{w}}$ and $\mathbf{q}$ for $\overline{\mathbf{q}}$.

Step A: Now the chain rule is effected on functions analogous to (8)-(9) and (16)-(17) and on functionals analogous to (13) and (21). Varying $g(\mathbf{q}, V, \hat{\mathbf{v}})=\bar{g}(\overline{\mathbf{q}}, A, \hat{\mathbf{w}})$ in the label 
(coordinates) dependence, and then equating as above, gives

$$
\begin{aligned}
& \frac{\partial g}{\partial \mathbf{q}}=\frac{\partial \bar{g}}{\partial \overline{\mathbf{q}}}-\frac{V_{B_{i}}}{V_{A}} \frac{\partial B_{i}}{\partial \mathbf{q}} \frac{\partial \bar{g}}{\partial A} \\
& \frac{\partial g}{\partial V}=\frac{1}{V_{A}} \frac{\partial \bar{g}}{\partial A} \\
& \frac{\partial g}{\partial \hat{\mathbf{v}}}=\frac{\partial \bar{g}}{\partial \hat{\mathbf{w}}}-\frac{1}{V_{A}} \frac{\partial V}{\partial \hat{\mathbf{v}}} \frac{\partial \bar{g}}{\partial A} .
\end{aligned}
$$

Inserting (26) and (27) into (16) gives the chain rule on functions

$$
\begin{aligned}
D_{*} \bar{g} & =\frac{\partial g}{\partial \mathbf{v}}=\frac{1}{V}\left(\frac{\partial \bar{g}}{\partial \hat{\mathbf{w}}}-\frac{1}{V_{A}} \frac{\partial \bar{g}}{\partial A} \frac{\partial V}{\partial \hat{\mathbf{w}}}\right) \cdot \overline{\overline{\mathcal{I}}}_{\perp}+\frac{\hat{\mathbf{w}}}{V_{A}} \frac{\partial \bar{g}}{\partial A} \\
& =\frac{1}{V} \partial_{\hat{\mathbf{w}}} \bar{g} \cdot \overline{\overline{\mathcal{I}}}_{\perp}+\frac{\partial_{A} \bar{g}}{V_{A}} \hat{\mathbf{w}}-\frac{\partial_{A} \bar{g}}{V V_{A}} \partial_{\hat{\mathbf{w}}} V \cdot \overline{\overline{\mathcal{I}}}_{\perp},
\end{aligned}
$$

while (25) gives

$$
\nabla_{*} \bar{g}=\frac{\partial g}{\partial \mathbf{q}}=\frac{\partial \bar{g}}{\partial \overline{\mathbf{q}}}-\frac{V_{B_{i}}}{V_{A}} \frac{\partial B_{i}}{\partial \overline{\mathbf{q}}} \frac{\partial \bar{g}}{\partial A}=\bar{\nabla} \bar{g}-\frac{V_{B_{i}}}{V_{A}} \bar{\nabla} B_{i} \partial_{A} \bar{g}
$$

Then, inserting (28) and (29) into (18) gives the following complicated expression for the particle bracket [, ] in the new coordinates:

$$
\begin{aligned}
{[\bar{g}, \bar{h}] } & =\nabla_{*} \bar{g} \cdot D_{*} \bar{h}-\nabla_{*} \bar{h} \cdot D_{*} \bar{g}+e \mathbf{B} \cdot\left(D_{*} \bar{g} \times D_{*} \bar{h}\right) \\
& =\frac{1}{V}\left(\bar{\nabla} \bar{g} \cdot \overline{\overline{\mathcal{I}}}_{\perp} \cdot \partial_{\hat{\mathbf{w}}} \bar{h}-\bar{\nabla} \bar{h} \cdot \overline{\overline{\mathcal{I}}}_{\perp} \cdot \partial_{\hat{\mathbf{w}}} \bar{g}\right) \\
& +\frac{\hat{\mathbf{w}}}{V_{A}} \cdot\left(\bar{\nabla} \bar{g} \partial_{A} \bar{h}-\bar{\nabla} \bar{h} \partial_{A} \bar{g}\right) \\
& +\frac{\partial_{\hat{\mathbf{w}}} V \cdot \overline{\overline{\mathcal{I}}}_{\perp}}{V V_{A}} \cdot\left(\bar{\nabla} \bar{h} \partial_{A} \bar{g}-\bar{\nabla} \bar{g} \partial_{A} \bar{h}\right) \\
& +\frac{V_{B_{i}}}{V V_{A}} \bar{\nabla} B_{i} \cdot \overline{\mathcal{I}}_{\perp} \cdot\left(\partial_{\hat{\mathbf{w}}} \bar{g} \partial_{A} \bar{h}-\partial_{\hat{\mathbf{w}}} \bar{h} \partial_{A} \bar{g}\right) \\
& +\frac{e \mathbf{B}}{V^{2}} \cdot\left(\partial_{\hat{\mathbf{w}}} \bar{g} \cdot \overline{\overline{\mathcal{I}}}_{\perp}\right) \times\left(\partial_{\hat{\mathbf{w}}} \bar{h} \cdot \overline{\overline{\mathcal{I}}}_{\perp}\right) \\
& +\frac{e \mathbf{B} \times \hat{\mathbf{w}}}{V V_{A}} \cdot\left(\partial_{\hat{\mathbf{w}}} \bar{h} \partial_{A} \bar{g}-\partial_{\hat{\mathbf{w}}} \bar{g} \partial_{A} \bar{h}\right) \\
& -\frac{e \mathbf{B}}{V^{2} V_{A}} \times\left(\overline{\mathcal{I}}_{\perp} \cdot \partial_{\hat{\mathbf{w}}} V\right) \\
& \cdot \overline{\overline{\mathcal{I}}}_{\perp} \cdot\left(\partial_{\hat{\mathbf{w}}} \bar{h} \partial_{A} \bar{g}-\partial_{\hat{\mathbf{w}}} \bar{g} \partial_{A} \bar{h}\right) .
\end{aligned}
$$

Step B: The Jacobian for this transformation is now

$$
\begin{aligned}
d z & =V^{2} d V d \Omega d^{3} q=V^{2} V_{A} d A d \Omega d^{3} q \\
& =\mathcal{J} d A d \Omega d^{3} q=: \mathcal{J} d \eta d^{3} q=: \mathcal{J} d w
\end{aligned}
$$


which define the Jacobian $\mathcal{J}$ and the integration measures $d \eta$ and $d w$. Note that these are not the same as those of Sec. IV, even though the same symbols are used. Furthermore, $\mathcal{J}$ now depends on $\mathbf{B}$ and, hence, q. Also, $d \Omega$ contains a portion of the Jacobian from cartesian coordinates, but one that is independent of $\mathbf{q}$.

Step C: Now consider the functional chain rule as above,

$$
\begin{aligned}
\delta F & =\int d z F_{f} \delta f+\int d^{3} q\left(F_{\mathbf{B}} \cdot \delta \mathbf{B}+F_{\mathbf{E}} \cdot \delta \mathbf{E}\right) \\
& =\int d w \bar{F}_{\bar{f}} \delta \bar{f}+\int d^{3} q\left(\bar{F}_{\overline{\mathbf{B}}} \cdot \delta \overline{\mathbf{B}}+\bar{F}_{\overline{\mathbf{E}}} \cdot \delta \overline{\mathbf{E}}\right),
\end{aligned}
$$

Functionally varying $f(\mathbf{q}, V, \hat{\mathbf{v}})=\bar{f}(\overline{\mathbf{q}}, A, \hat{\mathbf{w}})$ gives

$$
\delta f=\delta \bar{f}+\frac{\partial \bar{f}}{\partial A} \frac{\partial A}{\partial \mathbf{B}} \cdot \delta \mathbf{B}
$$

while $\delta \mathbf{B}=\delta \overline{\mathbf{B}}$ and $\delta \mathbf{E}=\delta \overline{\mathbf{E}}$. Whence, upon substitution of (32) into (31), the chain rule on functionals is obtained,

$$
\begin{aligned}
& \frac{\delta F}{\delta f}=\frac{1}{\mathcal{J}} \frac{\delta \bar{F}}{\delta \bar{f}}, \\
& \frac{\delta F}{\delta \mathbf{E}}=\frac{\delta \bar{F}}{\delta \overline{\mathbf{E}}}, \\
& \frac{\delta F}{\delta \mathbf{B}}=\frac{\delta \bar{F}}{\delta \overline{\mathbf{B}}}-\int d \eta \frac{\partial A}{\partial \mathbf{B}} \frac{\partial \bar{f}}{\partial A} \frac{\delta \bar{F}}{\delta \bar{f}},
\end{aligned}
$$

where the last expression of (33) can be written in a more convenient way as

$$
\frac{\delta F}{\delta \mathbf{B}}=\frac{\delta \bar{F}}{\delta \overline{\mathbf{B}}}+\int d \eta \frac{V_{\overline{\mathbf{B}}}}{V_{A}} \frac{\partial \bar{f}}{\partial A} \frac{\delta \bar{F}}{\delta \bar{f}} .
$$

This follows from

$$
\frac{\partial A}{\partial \mathbf{B}}=-\frac{V_{\overline{\mathbf{B}}}}{V_{A}}
$$

which comes about because the change in $A$ induced by a change in $\mathbf{B}$ at fixed $V$ and $\hat{\mathbf{w}}$, satisfies $0=\delta V=V_{A} \delta A+V_{\bar{B}_{i}} \delta \bar{B}_{i}$.

Finally, the Maxwell-Vlasov bracket in the coordinates $(\mathbf{q}, A, \hat{\mathbf{v}})$ is given by

$$
\begin{aligned}
& \{F, G\}=\int d \eta d^{3} q \mathcal{J} f\left[\mathcal{J}^{-1} F_{f}, \mathcal{J}^{-1} G_{f}\right] \\
& +e \int d \eta d^{3} q \mathcal{J} f\left(G_{\mathbf{E}} \cdot D_{*} \mathcal{J}^{-1} F_{f}-F_{\mathbf{E}} \cdot D_{*} \mathcal{J}^{-1} G_{f}\right) \\
& +\int d^{3} q\left(F_{\mathbf{E}} \cdot \nabla \times\left[G_{\mathbf{B}}+\int d \eta \frac{V_{\mathbf{B}}}{V_{A}} \frac{\partial f}{\partial A} \frac{\delta G}{\delta f}\right]\right. \\
& \left.-G_{\mathbf{E}} \cdot \nabla \times\left[F_{\mathbf{B}}+\int d \eta \frac{V_{\mathbf{B}}}{V_{A}} \frac{\partial f}{\partial A} \frac{\delta F}{\delta f}\right]\right),
\end{aligned}
$$


where the particle bracket [, ] is given by $(30), D_{*}$ is the operator defined by (28), and the bars have been dropped.

\section{LIFTING WITH NONLOCAL DEPENDENCE ON B}

In order to include the physical coordinates where $A$ is the magnetic moment $\mu$, the last step is to consider the case where the coordinate transformation involves derivatives of the magnetic field. This is important because perturbative reductions, such as those based on Lie-transforms [21-23] or mixed variable generating functions [24], often involve derivatives to arbitrary high order in the fields.

So, a more general transformation to new coordinates $(\mathbf{q}, V, \hat{\mathbf{v}}) \leftrightarrow(\overline{\mathbf{q}}, A, \hat{\mathbf{w}})$ is considered:

$$
\mathbf{q}=\overline{\mathbf{q}}, \quad \hat{\mathbf{v}}=\hat{\mathbf{w}}, \quad \text { and } \quad V=V[A, \hat{\mathbf{w}}, \mathbf{B}]
$$

where now $V[A, \hat{\mathbf{w}}, \mathbf{B}]$ means a transformation that depends on $\mathbf{B}$ and, possibly, all its derivatives. For an example of an allowable function $V$, see Sec. VII. Clearly, invertibility requires $V_{A}:=\partial V / \partial A \neq 0$, which is the only assumption on this function. Since the first two equations above are identities, as before eventually $\hat{\mathbf{v}}$ will be used for $\hat{\mathbf{w}}$ and $\mathbf{q}$ for $\overline{\mathbf{q}}$.

Step B: The Jacobian for this transformation is again

$$
d z=V^{2} V_{A} d A d \Omega d^{3} q=\mathcal{J} d A d \Omega d^{3} q=: \mathcal{J} d \eta d^{3} q=: \mathcal{J} d w
$$

but now $\mathcal{J}$ depends on $\mathbf{q}$ through $\mathbf{B}$ and its derivatives.

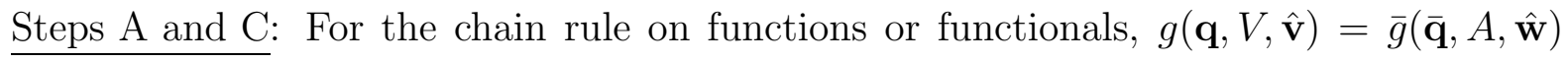
and $h(\mathbf{q}, V, \hat{\mathbf{v}})=\bar{h}(\overline{\mathbf{q}}, A, \hat{\mathbf{w}})$ are varied as in Sec. V, and all terms are the same as before, except some slight changes in the relations involving derivatives with respect to the magnetic field (Step D), which we study in the next paragraph.

Step D: Indeed, the Fréchet derivative with respect to $\mathbf{B}$ is now a differential operator, and care must be taken with the order of terms. For instance, relation (25) becomes

$$
\frac{\partial g}{\partial \mathbf{q}}=\frac{\partial \bar{g}}{\partial \overline{\mathbf{q}}}-\frac{\partial \bar{g}}{\partial A} \frac{1}{V_{A}} V_{B_{i}} \frac{\partial B_{i}}{\partial \mathbf{q}}
$$


where $V_{B_{i}}$ is now a differential operator acting on $\partial B_{i} / \partial \mathbf{q}$. Formulae (29)-(30) must be changed accordingly.

As for relations (32)-(33), variation is performed slightly differently this time as follows:

$$
\delta \bar{f}=\delta f+f_{V} V_{\overline{\mathbf{B}}} \cdot \delta \overline{\mathbf{B}}
$$

where $V_{\overline{\mathbf{B}}}$ is the Fréchet derivative operating on $\delta \overline{\mathbf{B}}$. Thus the chain rule for functional derivatives gives

$$
\frac{\delta F}{\delta \mathbf{B}}=\frac{\delta \bar{F}}{\delta \overline{\mathbf{B}}}+\int d \eta V_{\mathbf{B}}^{\dagger}\left(\frac{\partial f}{\partial V} \frac{\delta \bar{F}}{\delta \bar{f}}\right)=\frac{\delta \bar{F}}{\delta \overline{\mathbf{B}}}+\int d \eta V_{\mathbf{B}}^{\dagger}\left(\frac{\bar{F}_{\bar{f}}}{V_{A}} \frac{\partial \bar{f}}{\partial A}\right)
$$

where the adjoint $\dagger$ is done with respect to $d w$.

Finally, the Maxwell-Vlasov bracket (34) in these coordinates becomes

$$
\begin{aligned}
& \{F, G\}=\int d \eta d^{3} q \mathcal{J} f\left[\mathcal{J}^{-1} F_{f}, \mathcal{J}^{-1} G_{f}\right] \\
& +e \int d \eta d^{3} q \mathcal{J} f\left(G_{\mathbf{E}} \cdot D_{*} \mathcal{J}^{-1} F_{f}-F_{\mathbf{E}} \cdot D_{*} \mathcal{J}^{-1} G_{f}\right) \\
& +\int d^{3} q\left(F_{\mathbf{E}} \cdot \nabla \times\left[G_{\mathbf{B}}+\int d \eta V_{\mathbf{B}}^{\dagger}\left(\frac{G_{f}}{V_{A}} \frac{\partial f}{\partial A}\right)\right]\right. \\
& \left.-G_{\mathbf{E}} \cdot \nabla \times\left[F_{\mathbf{B}}+\int d \eta V_{\mathbf{B}}^{\dagger}\left(\frac{F_{f}}{V_{A}} \frac{\partial f}{\partial A}\right)\right]\right) .
\end{aligned}
$$

\section{AN EXAMPLE USING THE MAGNETIC MOMENT}

With the transformed bracket (37), the first thing to be checked is whether the dynamics agrees with the conservation of the magnetic moment, when appropriate, since this is what suggested the reduction in the first place. To this end, suppose the coordinate $A$ is the magnetic moment, $A:=\mu(\mathbf{q}, \mathbf{v})$, which to lowest order is given by $A=\left\|\mathbf{v}_{\perp}\right\|^{2} /\|\mathbf{B}\|$. To get a true conserved quantity, small corrections must be added to all orders in the Larmor radius, including derivatives of all orders in the magnetic field [16, 21].

This illustrates an example of an allowable function $V$. For the case studied in Sec. V, one can choose the coordinate $V(A)$ to be the inverse of the zeroth-order magnetic moment relation $A=\left\|\mathbf{v}_{\perp}\right\|^{2} /\|\mathbf{B}\|=V^{2}\|\hat{\mathbf{v}} \times \hat{\mathbf{b}}\|^{2} /\|\mathbf{B}\|$ :

$$
V_{0}:=\frac{\sqrt{A\|\mathbf{B}\|}}{\|\hat{\mathbf{v}} \times \hat{\mathbf{b}}\|}=\frac{\sqrt{A}\left(\mathbf{B}^{2}\right)^{3 / 4}}{\sqrt{(\hat{\mathbf{v}} \times \mathbf{B})^{2}}},
$$


which implies for the function $V_{\mathbf{B}}$ of formula (34)

$$
\left(V_{0}\right)_{\mathbf{B}}=\frac{3}{4} \frac{V}{\mathbf{B}^{2}}\left(\mathbf{B}^{2}\right)_{\mathbf{B}}-\frac{1}{2} \frac{V}{(\hat{\mathbf{v}} \times \mathbf{B})^{2}}\left((\hat{\mathbf{v}} \times \mathbf{B})^{2}\right)_{\mathbf{B}}=\frac{V}{2 B}\left(3 \hat{\mathbf{b}}-2 \frac{\overline{\overline{\mathcal{I}}}_{\perp} \cdot \hat{\mathbf{b}}}{(\hat{\mathbf{v}} \times \hat{\mathbf{b}})^{2}}\right) .
$$

For the case studied in Sec. VI, one can choose the coordinate $V(A)$ to be the inverse of following expression for the magnetic moment to first order [16, 21]:

$$
A=\frac{\left\|\mathbf{v}_{\perp}\right\|^{2}}{\|\mathbf{B}\|}-2 \frac{\left\|\mathbf{v}_{\perp}\right\|^{3}}{\|\mathbf{B}\|^{2}}\left[\frac{\mathrm{a} \cdot \nabla B}{2 B}+\phi \frac{3 \mathrm{a} \cdot \nabla \hat{\mathbf{b}} \cdot \mathbf{c}-\mathbf{c} \cdot \nabla \hat{\mathbf{b}} \cdot \mathbf{a}}{4}+\phi^{2} \hat{\mathbf{b}} \cdot \nabla \hat{\mathbf{b}} \cdot \mathbf{a}\right],
$$

where $\phi:=(\hat{\mathbf{b}} \cdot \hat{\mathbf{v}}) /\left(\sqrt{(\hat{\mathbf{b}} \cdot \hat{\mathbf{v}})^{2}}\right)$, and the vectors $\mathbf{a}:=(\hat{\mathbf{b}} \times \hat{\mathbf{v}}) /\|\hat{\mathbf{v}} \times \hat{\mathbf{v}}\|$ and $c:=\mathbf{a} \times \hat{\mathbf{b}}$ are the unit vectors of the perpendicular velocity and of the Larmor radius, respectively. In Eq. (38), the second term is assumed to be small compared to the first. Then, the inverse function $V(A)$ is given by

$$
V_{1}:=\frac{1}{\left\|\mathbf{v}_{\perp}\right\|}\left\{\sqrt{A B}+A\left[\frac{\mathrm{a} \cdot \nabla B}{2 B}+\phi \frac{3 \mathrm{a} \cdot \nabla \hat{\mathbf{b}} \cdot \mathrm{c}-\mathrm{c} \cdot \nabla \hat{\mathbf{b}} \cdot \mathbf{a}}{4}+\phi^{2} \hat{\mathbf{b}} \cdot \nabla \hat{\mathbf{b}} \cdot \mathbf{a}\right]\right\} .
$$

Here, we are not interested in computing the complete quantity $V_{\mathbf{B}}$, but only in showing how it can be an operator when the coordinate $V$ involves derivatives of the magnetic field. As an example, consider the term $\frac{A}{\left\|\mathbf{v}_{\perp}\right\|} \frac{\mathrm{a} \cdot \nabla B}{2 B}$ in (39). When computing $V_{\mathbf{B}}$, the contribution coming from $B$, which is acted upon by the gradient, is given by the first variation in $\delta \mathbf{B}$ of

$$
\frac{A \mathrm{a} \cdot \nabla}{2 B\left\|\mathbf{v}_{\perp}\right\|} \sqrt{(\mathbf{B}+\delta \mathbf{B})^{2}}-\frac{A \mathrm{a} \cdot \nabla}{2 B\left\|\mathbf{v}_{\perp}\right\|} \sqrt{\mathbf{B}^{2}}
$$

which is just

$$
\frac{A \mathrm{a} \cdot \nabla}{2 B\left\|\mathbf{v}_{\perp}\right\|}(\hat{\mathbf{b}} \cdot \delta \mathbf{B})=\frac{A}{2 B\left\|\mathbf{v}_{\perp}\right\|}(\mathbf{a} \cdot \nabla \hat{\mathbf{b}} \cdot+\hat{\mathbf{b}} \cdot(\mathbf{a} \cdot \nabla)) \delta \mathbf{B} .
$$

The corresponding contribution to $V_{\mathbf{B}_{i}}$ is

$$
\frac{A}{2 B\left\|\mathbf{v}_{\perp}\right\|}\left(\mathrm{a} \cdot \nabla \hat{\mathbf{b}}_{i}+\hat{\mathbf{b}}_{i} \mathbf{a} \cdot \nabla\right) .
$$

As expected, it is a first-order differential operator.

For a more precise expression of the magnetic moment, higher order corrections could be added, and $V_{\mathbf{B}}$ would be a differential operator of higher order.

Let us now check the conservation of the magnetic moment $A=\left\|\mathbf{v}_{\perp}\right\|^{2} /\|\mathbf{B}\|+O(\epsilon)$, which is exactly defined as solution of the following equation

$$
0=\dot{\mu}=\mathbf{v} \cdot \nabla \mu+e \mathbf{v} \times \mathbf{B} \cdot \partial_{\mathbf{v}} \mu .
$$


At the field level, the conservation of the magnetic moment corresponds to the conservation of the functional

$$
M:=\int d z f \mu
$$

for any particle distribution $f$. In the transformed coordinates, this is

$$
\bar{M}:=\int d w \mathcal{J} \bar{f} \mu .
$$

To investigate the conservation of $\bar{M}$, note that a static magnetic field corresponds to elimination of the electric field term in the Hamiltonian functional, since this eliminates the $\nabla \times \mathbf{E}$ term in the Maxwell-Faraday equation. In this case

$$
\begin{aligned}
\dot{\bar{M}} & =\{\bar{M}, \bar{H}\}=\int d \eta d^{3} q \mathcal{J} \bar{f}\left[\mu, \mathcal{J}^{-1} \bar{H}_{\bar{f}}\right] \\
& =\frac{1}{2} \int d \eta d^{3} q \mathcal{J} \bar{f}\left(\nabla_{*} \mu \cdot D_{*} V^{2}+e \mathbf{B} \cdot D_{*} \mu \times D_{*} V^{2}\right) \\
& =\int d^{3} v d^{3} q f\left(\mathbf{v} \cdot \nabla \mu+\mathbf{v} \times e \mathbf{B} \cdot \partial_{\mathbf{v}} \mu\right)=0,
\end{aligned}
$$

as was expected.

Accordingly, the transformed bracket (37) is expressed in coordinates adapted to the conserved magnetic moment. As is ususal in gyrokinetics, the electromagnetic field dynamics spoils the conservation of the magnetic moment. This is why the feed-back of the plasma dynamics onto the electromagnetic field dynamics needs to be restored as a perturbation, i.e., a perturbed magnetic moment must be defined that is conserved [17].

Consider now the transformed Maxwell-Vlasov equations of motion generated by the bracket (37). In this bracket, most of the terms are actually identical to those of the initial bracket (2), even though their formal expressions look different because they are expressed in the reduced coordinates $(\overline{\mathbf{q}}, A, \hat{\mathbf{w}})$, e.g. through formulae (28) and (30). The only new terms are

$$
\int d^{3} q \bar{F}_{\overline{\mathbf{E}}} \cdot \nabla \times \int d \eta V_{\overline{\mathbf{B}}}^{\dagger}\left(\frac{\bar{G}_{f}}{V_{A}} \frac{\partial \bar{f}}{\partial A}\right)
$$

and one obtained by permuting $\bar{F}$ and $\bar{G}$ (and with a minus sign for bracket antisymmetry).

In the equations of motion, this new bracket term generates an additional term in Maxwell-Ampere equation, viz.

$$
\begin{aligned}
\dot{\overline{\mathbf{E}}}=\nabla \times \bar{H}_{\overline{\mathbf{B}}}- & e \int d \eta \mathcal{J} \bar{f} D_{*}\left(\mathcal{J}^{-1} \bar{H}_{\bar{f}}\right) \\
& +\nabla \times \int d \eta V_{\overline{\mathbf{B}}}^{\dagger}\left(\frac{\bar{H}_{f}}{V_{A}} \frac{\partial \bar{f}}{\partial A}\right) .
\end{aligned}
$$


At first glance this additional term looks like a new magnetization current. But, one must remember that the usual $\nabla \times \mathbf{B}$ term has itself another additional contribution $\nabla \times \delta \bar{H}_{k i n} / \delta \overline{\mathbf{B}}$, because in the reduced variables, the plasma kinetic energy depends on the magnetic field $\bar{H}_{k i n}:=\int d w \mathcal{J} \bar{f} V^{2} / 2$ that is not constant in $\overline{\mathbf{B}}$ (both because of $\mathcal{J}$ and $V$ ). And, it turns out that this last additional contribution exactly cancels the "magnetization" term in (40):

$$
\begin{aligned}
\frac{\delta \bar{H}_{k i n}}{\delta \overline{\mathbf{B}}(\mathbf{x})} & =\frac{1}{2} \int d w \bar{f}\left(\mathcal{J} V^{2}\right)_{\overline{\mathbf{B}}} \delta(\mathbf{q}-\mathbf{x}) \\
& =\frac{1}{2} \int d w \bar{f}\left(\partial_{A} V \cdot V^{4}\right)_{\overline{\mathbf{B}}} \delta(\mathbf{q}-\mathbf{x}) \\
& =-\frac{1}{10} \int d w \partial_{A} \bar{f}\left(V^{5}\right)_{\overline{\mathbf{B}}} \delta(\mathbf{q}-\mathbf{x}) \\
& =-\frac{1}{10} \int d w \partial_{A} \bar{f}\left(V^{5}\right)_{V} V_{\overline{\mathbf{B}}} \delta(\mathbf{q}-\mathbf{x}) \\
& =-\frac{1}{2} \int d w \partial_{A} \bar{f} \mathcal{J} V^{2} V_{A} V_{\overline{\mathbf{B}}} \delta(\mathbf{q}-\mathbf{x}) \\
& =-\int d w \partial_{A} \bar{f} \frac{\bar{H}_{\bar{f}}}{V_{A}} V_{\overline{\mathbf{B}}} \delta(\mathbf{q}-\mathbf{x}) \\
& =-\int d \eta V_{\overline{\mathbf{B}}}^{\dagger}\left(\frac{\bar{H}_{\bar{f}}}{V_{A}} \partial_{A} \bar{f}\right) .
\end{aligned}
$$

This cancellation was to be expected, since the electric field $\mathbf{E}=\overline{\mathbf{E}}$ is not affected by the change of velocity coordinates, and the current term has not been changed either, but only expressed in the new variables:

$$
-e \int d \eta \mathcal{J} \bar{f} D_{*}\left(\mathcal{J}^{-1} \bar{H}_{\bar{f}}\right)=-\frac{e}{2} \int d^{3} v f \partial_{\mathbf{v}}\left(\mathcal{J}^{-1} \mathcal{J}\|\mathbf{v}\|^{2}\right)=-\mathbf{J}
$$

Finally, the additional term in the transformed bracket (37) generates another additional term in the equation of motion: the dynamics of the Vlasov phase space density $\dot{f}$ has an additional force term

$$
-\frac{1}{V_{A}} \frac{\partial \bar{f}}{\partial A} V_{\overline{\mathbf{B}}} \cdot \nabla \times \overline{\mathbf{E}}
$$

This term is not cancelled by any other term. It can be rewritten as

$$
-\frac{\partial f}{\partial V} V_{\mathbf{B}} \cdot \nabla \times \mathbf{E}=\frac{\partial f}{\partial V} V_{\mathbf{B}} \cdot \dot{\mathbf{B}}
$$

which is exactly the expected contribution when applying the chain rule for the time derivative of the transformed fields. It comes about because the change of coordinates is timedependent when the magnetic field is not static. 


\section{CONCLUSION}

In summary, in this paper techniques for transforming the Vlasov-Maxwell Poisson bracket to new coordinates, when the transformation law mixes dependent and independent variables, have been developed. Four transformations were considered, each of which considered a new feature needed for understanding the more general transformation of (35). In Sec. III a transformation that mixed the independent velocity variable with the magnetic field was considered and the associated function and functional chain rules were described. In Sec. IV, spherical velocity coordinates were treated and here it was seen how a nontrivial Jacobian determinant influences a transformation. In Sec. V a class of transformations that mixes the dependent and independent variables by having dependence on $\mathbf{B}$ and in addition possesses a nontrivial Jacobian was considered. Finally, in Sec. VI, the nonlocal transformation of (35) was effected, the most general transformation of this paper that results in the transformed noncanonical Poisson bracket of (37). This final form of the Poisson bracket was seen to contain additional terms that appear to be magnetization-like contributions. However, these bracket terms were shown to produce no magnetization term in the equations of motion, since the electromagnetic fields are not affected by the change of field coordinates. Only the dynamics of the Vlasov density obtained an additional term, a term that results from the change of field coordinates being time-dependent through $\mathbf{B}$.

The transformations of Secs. III-VI paved the way for the simple example of Sec. VII. Here the dynamics was reduced by dropping the electric field energy from the Hamiltonian, resulting in the magnetic moment being conserved by a reduced dynamics that must have a static magnetic field. However, when restoring the feed-back of the plasma dynamics onto the electromagnetic field dynamics, the magnetic moment was seen to be no longer conserved and must be perturbatively changed to be conserved.

In all the cases considered, the lifting was eased because the change of coordinates only concerned a new particle velocity that depends on the magnetic field, but no change was made in the spatial coordinate. If Eq. (1) is generalized by adding dependence on the electric field and all its derivatives, then results similar to those presented are immediate. However, if the new spatial variable has velocity and field dependence, then the situation becomes considerably more complex. Such transformations are of interest for some oscillation-center, guiding-center, and gyrokinetic theory development, and the same methods of function and 
functional chain rule can be used, but some additional effects will show up, e.g., non-zero polarization and magnetization terms like those of [12]. Details of the magnetic moment reduction will be given in [21] and more general lifting will be considered in a future publication.

\section{ACKNOWLEDGMENT}

We acknowledge financial support from the Agence Nationale de la Recherche (ANR GYPSI). This work was also supported by the European Community under the contract of Association between EURATOM, CEA, and the French Research Federation for fusion study. The views and opinions expressed herein do not necessarily reflect those of the European Commission. PJM was supported by U.S. Department of Energy contract \# DEFG05-80ET-53088. The authors also acknowledge fruitful discussions with Alain Brizard, and with members of the Équipe de Dynamique Nonlinéaire of the Centre de Physique Théorique of Marseille.

[1] C. L. Siegel and J. K. Moser, Lectures on Celestial Mechanics (Springer Verlag, Berlin, 1971).

[2] M. Born, The Mechanics of the Atom (F. Ungar, New York, 1967).

[3] M. Kruskal, J. Math. Phys. 3, 806 (1962).

[4] R. de la Llave, Introduction to KAM theory, in Computational Physics (World Scientic, River Edge, NJ, 1992) pp. 73-105.

[5] J. Henrard, The adiabatic invariant in classical dynamics, Dynamics Reported 2, (Springer, Berlin, 1993) p. 117 (new series).

[6] P. J. Morrison and J. M. Greene, Phys. Rev. Lett. 45, 790, (1980); E 48, 569 (1982).

[7] P. J. Morrison, AIP Conf. Proc. 88, 13 (1982).

[8] P. J. Morrison, Rev. Mod. Phys. 70, 467 (1998).

[9] P. J. Morrison, Phys. Lett. 80A, 383 (1980).

[10] A. Weinstein and P. J. Morrison, Phys. Lett. A 86, 235 (1981).

[11] J. E. Marsden and A. Weinstein, Physica D 4, 394 (1982).

[12] P. J. Morrison, Phys. Plasmas 20, 012104 (2013). 
[13] E. A. Frieman and L. Chen, Phys. Fluids 25, 502 (1982).

[14] R. G. Littlejohn, Phys. Fluids 27, 976 (1984).

[15] A. J. Brizard and A. Mischenko, J. Plas. Phys. 75, 675 (2009).

[16] J. R. Cary and A. J. Brizard, Rev. Mod. Phys. 81, 693 (2009).

[17] A. J. Brizard and T. S. Hahm, Rev. Mod. Phys. 79, 421 (2007).

[18] P. J. Morrison, Phys. Plasmas 12, 058102 (2005).

[19] J. Squire, H. Qin, W. M. Tang, and C. Chandre, "The Hamiltonian structure and EulerPoincaré formulation of the Vlasov-Maxwell and gyrokinetic systems," Phys. Plasmas in press (2013).

[20] H. Ye and P. J. Morrison, Phys. Fluids B 4, 771 (1992).

[21] L. de Guillebon, N. Tronko, M. Vittot, and Ph. Ghendrih, "Dynamical reduction for charged particles in a strong magnetic field without guiding-center", in preparation.

[22] J. R. Cary, Phys. Reports 79, 129 (1981).

[23] R. G. Littlejohn, J. Math. Phys. 23, 742 (1982).

[24] D. Correa-Restrepo and D. Pfirsch, J. Plasma Phys. 71, 1 (2005). 